BULLETIN OF THE

AMERICAN MATHEMATICAL SOCIETY

Volume 81, Number 3, May 1975

\title{
ON THE NORM FORM OF A FINITE GALOIS EXTENSION OVER Q
}

\author{
BY JIH-MIN SHYR ${ }^{1}$
}

Communicated by Robert Fossum, February 13, 1975

1. Introduction. Let $\lambda: T \rightarrow T^{\prime}$ be a $Q$-isogeny of algebraic tori defined over $\mathbf{Q}$, the rational number field. Then the isogeny $\lambda$ induces naturally the following maps (cf. [2]):

$$
\lambda_{v}: T_{v} \rightarrow T_{v}^{\prime}, \quad \lambda_{v}^{c}: T_{v}^{c} \rightarrow T_{v}^{\prime c}, \quad \lambda_{\mathbf{Q}}^{\infty}: T_{\mathrm{Q}}^{\infty} \rightarrow T_{\mathbf{Q}}^{\prime \infty}, \quad(\hat{\lambda})_{\mathbf{Q}}:\left(\hat{T}^{\prime}\right)_{\mathbf{Q}} \rightarrow(\hat{T})_{\mathbf{Q}}
$$

For a homomorphism $\alpha: G \rightarrow G^{\prime}$ of commutative groups with finite kernel and cokernel, we define the $q$-symbol of $\alpha$ by $q(\alpha)=[\operatorname{Cok} \alpha] /[\operatorname{Ker} \alpha]$. Then the $q$-symbols of the above maps are defined, and $q\left(\lambda_{v}^{c}\right)=1$ for almost all finite prime $v$; more precisely, if $K$ is a finite splitting field for $T$ and $T^{\prime}$ over $Q$, then $q\left(\lambda_{v}^{c}\right)=1$ whenever $v$ is prime to the degree of $\lambda$ and is unramified relative to $K / Q$. In [2] , we prove

THEOREM 1. The relative class number $h_{T} / h_{T^{\prime}}$ of $T, T^{\prime}$ over $\mathbf{Q}$ can be expressed as

$$
\frac{h_{T}}{h_{T^{\prime}}}=\frac{\tau_{T}}{\tau_{T^{\prime}}} \cdot \frac{q\left(\lambda_{\infty}\right)}{q\left(\lambda_{Q}^{\infty}\right) q\left((\hat{\lambda})_{Q}\right)} \cdot \prod_{v \neq \infty} q\left(\lambda_{v}^{c}\right)
$$

where $\tau_{T}$ (resp. $\tau_{T^{\prime}}$ ) is the Tamagawa number of $T$ (resp. $T^{\prime}$ ) over $\mathbf{Q}$.

In this paper, we apply Theorem 1 to the study of the norm form of a finite Galois extension over $\mathbf{Q}$.

2. Main theorem. Let $K / Q$ be a Galois extension of finite degree $n$. Denote by $N$ the norm map $R_{K / Q}\left(G_{m}\right) \rightarrow \mathbf{G}_{m}$, where $\mathbf{G}_{m}$ is the multiplicative group of the universal domain $\Omega$, and $R_{K / Q}$ is the Weil functor of restricting the field of definition from $K$ to $Q$ (cf. [3]). We have an exact sequence

AMS (MOS) subject classifications (1970). Primary 10C10, $20 \mathrm{G} 30$.

1 This paper is based on a part of the author's Ph.D. thesis, written at Johns Hopkins University under the direction of Professor T. Ono. For the unexplained notions, see [2]. 


$$
0 \rightarrow \operatorname{Ker} N \stackrel{i}{\rightarrow} R_{K / Q}\left(\mathbf{G}_{m}\right) \stackrel{N}{\longrightarrow} \mathbf{G}_{m} \rightarrow 0
$$

of tori defined over $\mathbf{Q}$, where $i$ is the canonical inclusion. We attach to $(\mathrm{N})$ a Q-isogeny $\lambda: R_{K / Q}\left(\mathbf{G}_{m}\right) \rightarrow \operatorname{Ker} N \times \mathbf{G}_{m}$ defined by $\lambda(x)=\left(x^{n} N(x)^{-1}\right.$, $N(x)$ ). Applying Theorem 1 to the isogeny $\lambda$, we obtain

THEOREM 2. Let $K, \lambda$ be as above. Then we have

$$
h_{K}=\frac{h_{1}}{\tau_{1}} \cdot \frac{q\left(\lambda_{\infty}\right)}{q\left(\lambda_{Q}^{\infty}\right) q\left((\hat{\lambda})_{Q}\right)} \cdot \prod_{v \neq \infty} q\left(\lambda_{v}^{c}\right),
$$

where $h_{K}$ is the class number of $K$, and $h_{1}$ (resp. $\left.\tau_{1}\right)$ is the class number (resp. the Tamagawa number) of the torus Ker $N$ over $\mathbf{Q}$.

Let $\left\{x_{1}, \ldots, x_{n}\right\}$ be an integral basis of $K$. The form $f$ defined by

$$
f\left(X_{1}, \ldots, X_{n}\right)=N_{K / Q}\left(x_{1} X_{1}+\cdots+x_{n} X_{n}\right)
$$

is an integral form in $n$ variables of degree $n$. The general linear group $\mathrm{GL}_{n}(\Omega)$ acts on the set of forms in $n$ variables as follows: if $u \in \mathrm{GL}_{n}(\Omega)$ and $g$ is a form in $n$ variables, then $(g u)\left(X_{1}, \ldots, X_{n}\right)=g\left(Y_{1}, \ldots, Y_{n}\right)$ with $\left(Y_{1}, \ldots, Y_{n}\right)^{t}=u\left(X_{1}, \ldots, X_{n}\right)^{t}$. We identify the torus $R_{K / \mathbf{Q}}\left(\mathbf{G}_{m}\right)$ with a subgroup of $\mathrm{GL}_{n}(\Omega)$ by means of the basis $\left\{x_{1}, \ldots, x_{n}\right\}$. Two integral forms $g, g^{\prime}$ in $n$ variables are said to be in the same $K$-class if $g^{\prime}=g z$ with $z$ in the set $R_{K / \mathbf{Q}}\left(\mathbf{G}_{m}\right)_{\mathbf{Z}}$ of elements of $R_{K / \mathbf{Q}}\left(\mathbf{G}_{m}\right) \cap M_{n}(\mathbf{Z})^{x}$. Also, $g, g^{\prime}$ are said to be in the same $K$-genus if $g^{\prime}=g t$ with $t$ in the set $R_{K / \mathbf{Q}}\left(\mathbf{G}_{m}\right)_{\mathbf{Q}}$ of elements of $R_{K / \mathbf{Q}}\left(\mathbf{G}_{m}\right)$ with rational coefficients, and $g^{\prime}=g u_{v}$ with $u=\left(u_{v}\right)_{v} \in R_{K / Q}\left(\mathbf{G}_{m}\right)_{\mathrm{A}}^{\infty}=R_{K / Q}\left(\mathbf{G}_{m}\right)_{\infty} \times \Pi_{v \neq \infty} R_{K / \mathbf{Q}}\left(\mathbf{G}_{m}\right)_{\mathbf{Z}_{v}}$, where $R_{K / Q}\left(\mathbf{G}_{m}\right)_{v}$ (resp. $R_{K / Q}\left(\mathbf{G}_{m}\right)_{\mathbf{z}_{v}}$ ) denotes the set of elements of $R_{K / \mathrm{Q}}\left(\mathbf{G}_{m}\right)$ with coefficients in $\mathbf{Q}_{v}$ (resp. $\left.R_{K / Q}\left(G_{m}\right) \cap M_{n}\left(\mathbf{Z}_{v}\right)^{x}\right)$. Let $H$ denote the kernel of the norm map $N: R_{K / Q}\left(\mathbf{G}_{m}\right) \rightarrow \mathbf{G}_{m}$.

MAIN THEOREM. There exists an injection $\Psi$ of the set of $K$-classes in the K-genus of $f$ into the quotient space $H_{\mathbf{A}} / H_{\mathbf{A}}^{\infty} \cdot H_{\mathbf{Q}}$. Moreover, if the class number of $K$ equals 1 , then $\Psi$ is a bijection and the number of $K$-classes in the $K$-genus of $f$ is given by

$$
\tau_{1} \cdot q\left(\lambda_{\mathbf{Q}}^{\infty}\right) q\left((\hat{\lambda})_{\mathbf{Q}}\right) / q\left(\lambda_{\infty}\right) \Pi_{p} q\left(\lambda_{p}^{c}\right)
$$

$\tau_{1}$ and the q-symbols being as in Theorem 2.

SKETCH OF THE PROOF. Take a $K$-class $[g]$ in the $K$-genus of $f$. By definition, we have $g=f t$ with $t \in R_{K / Q}\left(\mathbf{G}_{m}\right)_{\mathbf{Q}}$, and $g=f u_{v}$ with $u=\left(u_{v}\right)_{v}$ 
$\in R_{K / Q}\left(G_{m}\right)_{\mathbf{A}}$. This implies that $f=f u_{v} t^{-1}$ for all $v$. Putting $s_{v}=u_{v} t^{-1}$, we have $s_{v} \in H_{v}$ for all $v$, and $s_{v} \in H_{\mathbf{z}_{v}}$ for almost all finite prime $v$. Hence, $s=\left(s_{v}\right) \in H_{\mathrm{A}}$. We verify that the map defined by $\Psi([g])=s\left(H_{\mathrm{A}}^{\infty} \cdot H_{\mathrm{Q}}\right)$, is the desired injection. Furthermore, suppose that the class number of $K$ is 1 , i.e., $R_{K / \mathrm{Q}}\left(\mathbf{G}_{m}\right)_{\mathrm{A}}=R_{K / \mathrm{Q}}\left(\mathbf{G}_{m}\right)_{\mathrm{A}}^{\infty} \cdot R_{K / \mathrm{Q}}\left(\mathbf{G}_{m}\right)_{\mathrm{Q}}$. Take any $\operatorname{coset} s\left(H_{\mathrm{A}}^{\infty} \cdot H_{\mathrm{Q}}\right)$ in $H_{\mathrm{A}} / H_{\mathrm{A}}^{\infty} \cdot H_{\mathrm{Q}}$. Since $s=\left(s_{v}\right)_{v} \in H_{\mathrm{A}} \subset R_{K / \mathrm{Q}}\left(\mathrm{G}_{m}\right)_{\mathrm{A}}$, we can write $s=u t$ with $u=\left(u_{v}\right) \in R_{K / \mathrm{Q}}\left(\mathbf{G}_{m}\right)_{\mathrm{A}}^{\infty}$ and $t \in R_{K / \mathrm{Q}}\left(\mathbf{G}_{m}\right)_{\mathrm{Q}}$, i.e., $s_{v}=u_{v} t$ for all $v$. Then, $f=f s_{v}=f u_{v} t$ because $s=\left(s_{v}\right)_{v} \in H_{\mathrm{A}}$. From this follows that the $K$ class of the form $g$ defined by $g=f u_{v}=f t^{-1}$ is in the $K$-genus of $f$, and $\Psi([g])=s\left(H_{\mathrm{A}}^{\infty} \cdot H_{\mathrm{Q}}\right)$. The last assertion is an immediate consequence of Theorem 2.

REMARK. If $K$ is a finite abelian extension over $\mathbf{Q}$, the number $\tau_{1}$. $q\left(\lambda_{\mathrm{Q}}^{\infty}\right) q\left((\hat{\lambda})_{\mathrm{Q}}\right) / q\left(\lambda_{\infty}\right) \Pi_{p} q\left(\lambda_{p}^{c}\right)$ can be effectively computed by means of results in class field theory (cf. [2]). For example, if $K=\mathrm{Q}(\sqrt{m})$ is a quadratic field, we have $\tau_{1}=2$ (cf. [1]), $q\left((\hat{\lambda})_{\mathrm{Q}}\right)=1, q\left(\lambda_{\infty}\right)=1$,

$$
q\left(\lambda_{\mathbf{Q}}^{\infty}\right)= \begin{cases}2 & \text { if } m<0, \text { or } m>0 \text { and } N_{K / Q}(\epsilon)=-1, \\ 4 & \text { if } m>0 \text { and } N_{K / Q}(\epsilon)=1,\end{cases}
$$

where $\epsilon$ is a fundamental unit in $K$, and $\Pi_{p} q\left(\lambda_{p}^{c}\right)=2^{t+1}$, where $t$ is the number of distinct prime factors of the discriminant $d_{K}$ of $K$.

\section{REFERENCES}

1. T. Ono, On the Tamagawa number of algebraic tori, Ann. of Math. (2) 78 (1963), 47-73. MR 28 \#94.

2. J. Shyr, Class number formulas of algebraic tori with applications to relative class numbers of certain relative quadratic extensions of algebraic number fields, Ph.D. thesis, Johns Hopkins University, Baltimore, Md., 1974.

3. A. Weil, Adeles and algebraic groups, Lecture notes, Princeton University, Princeton, N. J., 1961.

DEPARTMENT OF MATHEMATICS, JOHNS HOPKINS UNIVERSITY, BALTIMORE, MARYLAND 21218 